of ionization by radioactive gamma rays is therefore different from that by cosmic radiation at sea-level, so much so that this behaviour may be utilized in distinguishing between gamma and cosmic rays ${ }^{3}$.

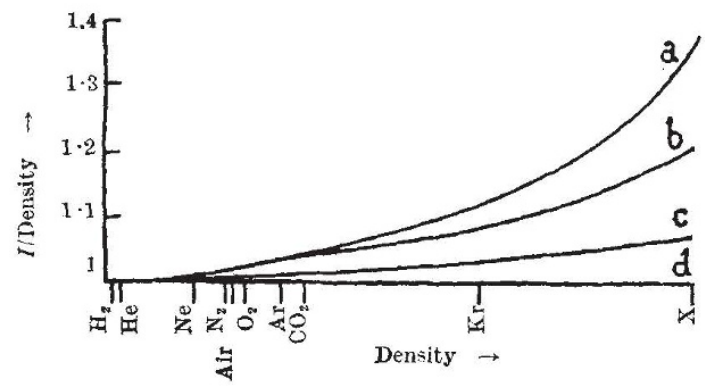

Fig. 1.

The ratio $I /$ Density plotted against Density. $a$, UNFILTERED PENETRATING RADIATION ; $b$, RADIUM C GAMMA RAYS FILTERED BY 6 MM. LEAD; $c$, COSMIC RAYS FILTERED BY 5 CM. LEAD; $d$, COSMIC RAYS FILTERED BY 10 CM. LEAD.

A short time ago Ziemecki ${ }^{4}$ gave the following results for the ratio of ionization by radioactive gamma rays in nitrogen, argon and krypton to air :

$$
I_{\mathrm{N}_{2}}: I_{\mathrm{Ar}}: I_{\mathrm{Kr}}=0 \cdot 49: 1: 2 \cdot 66 ;
$$

while the densities are

$$
\mathrm{PN}_{2}: \rho_{\mathrm{Ar}}: \mathrm{PKr}_{\mathrm{K}}=0 \cdot 77: 1: 2 \cdot 06 .
$$

These data for argon and krypton at 12 atm. agree with our results for one atmosphere $\left\langle I_{\mathrm{Ar}}: I_{\mathrm{Kr}}=\right.$ $1: 2 \cdot 28$ ) showing the same behaviour. However, Ziernecki believes the effects of ionization by gamma and cosmic rays to be roughly proportional. Our numerous measurements show only the hard com. ponents of the cosmic radiation at sea-level causing ionization in the ratio of the density of the gases examined.

Institute for Cosmic Ray Research,

J. JUULF.

University of Berlin,

Berlin-Dahlem.

Sept. 15.

'Masuch, V., Z. Phys., 79, 264 (1932).

${ }^{2}$ Juilfs, J. and Masuch, V., Z. Phys., 104, 458 (1937).

3 Juilfs, J., Phys. Z., 88, 691 (1937).

* Ziemecki, St., NaTure, 140, 150 (1937).

\section{Two Spectrometers for X-Ray Analysis}

Sometrmes a conical camera has been used to obtain good pictures of high-order layer-lines from a rotating crystal, the angle of the cone in most cases being about $60^{\circ}$ or $90^{\circ}$.

In several respects this method is awkward. By making that angle $180^{\circ}$, the difficulties will be less; the camera becomes more simple, the film easier to handle, and the identification of reflections will be more readily surveyed, every layer-line being a circle. Only the 0 - and perhaps one or more of the lower order layer-lines will be missing on that photograph.

It is possible to take a photograph on a stationary film and on a moving film at the same time. Another possibility is to couple the movement of the film in some manner to that of the crystal, for example, by moving the film parallel to the crystal rotation axis (but not around that axis). In this way it will always be possible to assign the appropriate indexes to the spots on the film.

A simple camera design of this type is shown in Fig. 1 ; a circular stationary film, $a$; another film at the same distance from the crystal, but rotating with the crystal, $b$; whereas the 0-layer-line is recorded on a narrow cylindrical film, $c$.

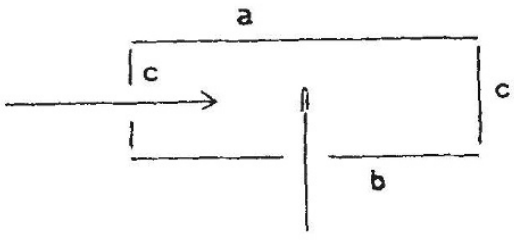

Fig. 1 .

For the investigation of powders it may be ad. vantageous to use camera with the film cylinder having the same axis as the $\mathrm{X}$-ray beam (see Fig. 2). The reflected beams, lying on cones, will cut the cylindrical film in circles; when spreading out the film after developing, the picture of straight lines will resemble a diagram taken with an ordinary optical spectrograph. Especially for purposes of identification, as well as for back-reflection X-ray

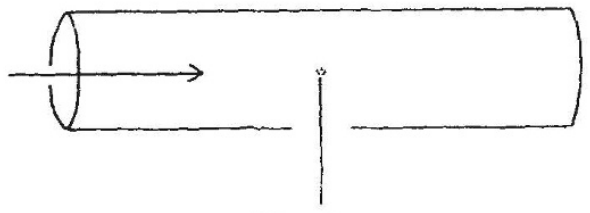

Fig. 2.

photographs, the method described may be useful. Taking a series of exposures on the same film, a narrow strip may be taken parallel to the primary beam. In that case a flat film or plate can be used.

I am indebted to Prof. Ir. J. A. Grutterink, who enabled me to design the cameras described.

Laboratorium voor Delfstofkunde der W. F. DE JONG. Technische Hoogeschool, Delft. Sept. 20.

\section{Spacing of the Resonance Neutron Levels of} Silver, Rhodium and Bromine Nuclei

From recent work on group and thermal neutrons ${ }^{1}$, the constants describing the resonance absorption of an element can be calculated fairly accurately. This simplifies the estimation of the average spacing of the resonance neutron levels in the experiment of Chao and $\mathrm{Fu}^{2}$ and makes it possible to work with a thinner paraffin scatterer so as to obtain a beam of neutrons distributed more uniformly over the energy spectrum. We have now made measurements with a paraffin scatterer of only $2 \mathrm{~mm}$. thickness. The neutron source consisted of $100 \mathrm{mgm}$. radium sur. rounded with $12 \mathrm{gm}$. beryllium, and the scatterer in the form of a spherical shell was of $4 \mathrm{~cm}$. outer diameter. Detectors of silver, rhodium and bromine, approximately $3 \mathrm{~cm} . \times 3 \mathrm{~cm}$. in size, were exposed at a distance of $1.2 \mathrm{~cm}$. from the paraffin, and the induced activity was measured with a Geiger-Müller counter. 